\title{
MAGNETIC ORDERING IN THE T* PHASE $\mathrm{La}_{1.2} \mathrm{~Tb}_{0.8} \mathrm{CuO}_{4}$
}

\section{A. LAPPAS and K. PRASSIDES}

School of Chemistry and Molecular Sciences, University of Sussex, Brighton $B N 19 Q J, U . K$.
A. AMATO, R. FEYERHERM, F.N. GYGAX and A. SCHENCK
Institute for Intermediate Energy Physics, Swiss Federal Institute of Technology (ETH) Zürich, CH-5232 Villigen PSI, Switzerland

We report a muon spin relaxation study of the magnetic properties of the $\mathrm{La}_{1.2} \mathrm{~Tb}_{0.8} \mathrm{CuO}_{4}$ phase with the $\mathrm{T}^{*}$ structure. Random magnetic order is revealed between 280 and $170 \mathrm{~K}$ by the zero field data. A spontaneous muon precession then appears below $170 \mathrm{~K}$, arising from antiferromagnetic long range order of the $\mathrm{Cu}^{2+}$ spins. Evidence exists below $20 \mathrm{~K}$ for ordering of the $\mathrm{Tb}^{3+}$ ions. We find that the $\mathrm{T}^{*}$ phase adopts the same magnetic structure as the $(\mathrm{T} / \mathrm{O})$ phase $\mathrm{La}_{2} \mathrm{CuO}_{4}$.

\section{Introduction}

The intimate relationship between the magnetic and (super)conducting properties of the high- $T_{c}$ materials has attracted much interest in recent years. Central to the discussion of superconductivity has been the evolution in the magnetic properties of the $\mathrm{CuO}_{2}$ layers since the antiferromagnetic (AF) long range order found for the undoped parent materials is always removed upon electron- or hole-doping before the onset of superconductivity. The $\mu \mathrm{SR}$ technique has been used extensively to study the magnetic phase diagrams of many families of cuprates, including $\mathrm{La}_{2-x} \mathrm{Sr}_{x} \mathrm{CuO}_{4}$ [1], $\mathrm{Nd}_{2-x} \mathrm{Ce}_{x} \mathrm{CuO}_{4}$ [2], $\mathrm{Nd}_{2-x} \mathrm{Sr}_{x} \mathrm{CuO}_{4}$ [3], $\mathrm{Y}_{1-x} \mathrm{Ln}_{x} \mathrm{Ba}_{2} \mathrm{Cu}_{3} \mathrm{O}_{7-\delta}$ [4], and $\mathrm{Bi}_{2} \mathrm{Sr}_{2} \mathrm{CaCu}_{2} \mathrm{O}_{8+\delta}$ [5]. Here we describe our recent muon spin relaxation measurements on $\mathrm{La}_{1.2} \mathrm{~Tb}_{0.8} \mathrm{CuO}_{4+\delta}$, the parent member of the $T^{*}$ structural family, in the temperature range 2 to $295 \mathrm{~K}$. Very little is currently known about the magnetic phase diagram in these systems in which $\mathrm{Cu}$ has a coordination number of 5 and which become superconducting on hole-doping [6]. Our results supplement the conclusions of recent magnetic neutron scattering measurements performed by our group [7] on the same samples. 


\section{Experimental}

The $\mathrm{La}_{1.2} \mathrm{~Tb}_{0.8} \mathrm{CuO}_{4+\delta}$ sample was synthesised by a sol-gel preparative route with all pellet firings performed in an oxygen flow [7]. Phase purity was established by $\mathrm{X}$-ray powder diffraction. Thermogravimetric analysis led to a value for the excess oxygen of $\delta=0.05$. The crystal structure was later refined using high-resolution powder neutron diffraction; it is found to remain strictly tetragonal in the whole temperature range of the present experiment [7]. Zero field (ZF) $\mu \mathrm{SR}$ experiments were performed in longitudinal geometry using both the pulsed muon facility spectrometer $(10-295 \mathrm{~K})$ at the Rutherford Appleton Laboratory (RAL) and the General Purpose Spectrometer (2.5 - $280 \mathrm{~K}$ ) at the Paul Scherrer Institute (PSI).

\section{Results}

Typical zero field spectra collected at RAL are shown in Fig. 1(a) for temperatures between 10 and $295 \mathrm{~K}$. The data were fitted throughout the temperature range using two Lorentzian components, one rapidly $\left(\lambda_{1}\right)$ and one slowly $\left(\lambda_{2}\right)$ relaxing. The normalised total asymmetry at $t=0$ shows a pronounced decrease below $\sim 170 \mathrm{~K}$ and then remains essentially constant below $\sim 110 \mathrm{~K}$. At the same time, the relaxation rate of the fast relaxing component shows a divergent character at the same temperature
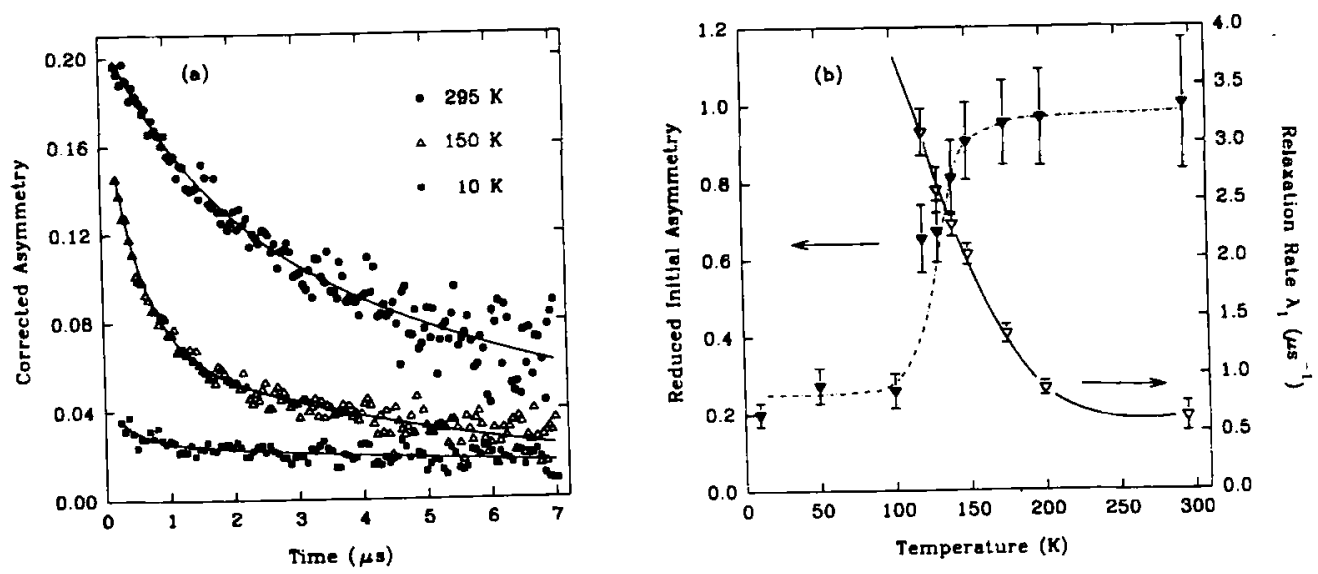

Fig. 1. (a) Zero field $\mu \mathrm{SR}$ spectra (RAL) of $\mathrm{La}_{1.2} \mathrm{~Tb}_{0.8} \mathrm{CuO}_{4+\delta}$ at 10,150 and $295 \mathrm{~K}$ fitted to two Lorentzian decays. (b) Temperature dependence of the normalised initial total asymmetry and the relaxation rate of the rapidly decaying component, $\lambda_{1}$. 
range where the asymmetry decreases (Fig. 1(b)). These results provide tentative evidence for a transition to a magnetically ordered state below $170 \mathrm{~K}$. In the low temperature range, it appears that we essentially observe only the fraction of the muons (approximately 'one-third') whose spins are parallel to the internal field and thus they do not precess.

Fig. 2 shows the time evolution of the muon spin polarisation of $\mathrm{La}_{1.2} \mathrm{~Tb}_{0.8} \mathrm{CuO}_{4+\delta}$ collected at PSI in zero external field at $75 \mathrm{~K}$. These superior data at early times can now be fitted with one oscillating (\#1) and one non-oscillating (\#2) Lorentzian component. Alternative model fitting functions were exhaustively eliminated. The ZF spontaneous muon precession frequency, $\nu_{1}$ approaches zero in the vicinity of $160-170 \mathrm{~K}$ which corresponds to the onset of the coherent ordering of the $\mathrm{Cu}$ magnetic moments (Fig. 3). However, component \#1 is still present at $\mathrm{T}>$ $170 \mathrm{~K}$, but its shape has changed to a Gaussian-damped non-oscillating signal whose amplitude decreases with increasing temperature, reaching a minimum value of $\sim 3 \%$ at $280 \mathrm{~K}$ (Fig. 2). Finally, at very low temperatures $(\mathrm{T}<20 \mathrm{~K})$ the depolarisation rate $\lambda_{1}$ increases dramatically reaching a value of $\sim 50 \mu \mathrm{s}^{-1}$. We take this as evidence for the onset of ordering of the moments of the rare-earth $\mathrm{Tb}^{3+}$ ions.

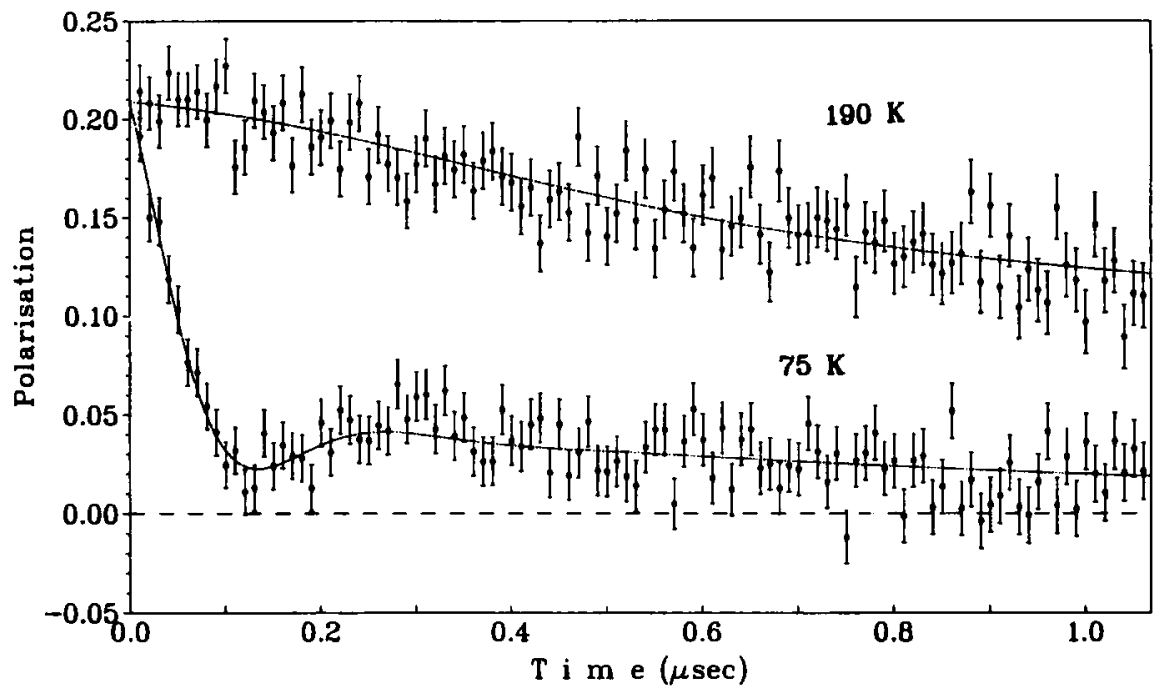

Fig. 2. Zero field $\mu \mathrm{SR}$ spectra (PSI) of $\mathrm{La}_{1.2} \mathrm{~Tb}_{0.8} \mathrm{CuO}_{4+\delta}$ at 75 and $190 \mathrm{~K}$. The solid curves are the two-component fits discussed in the text. 


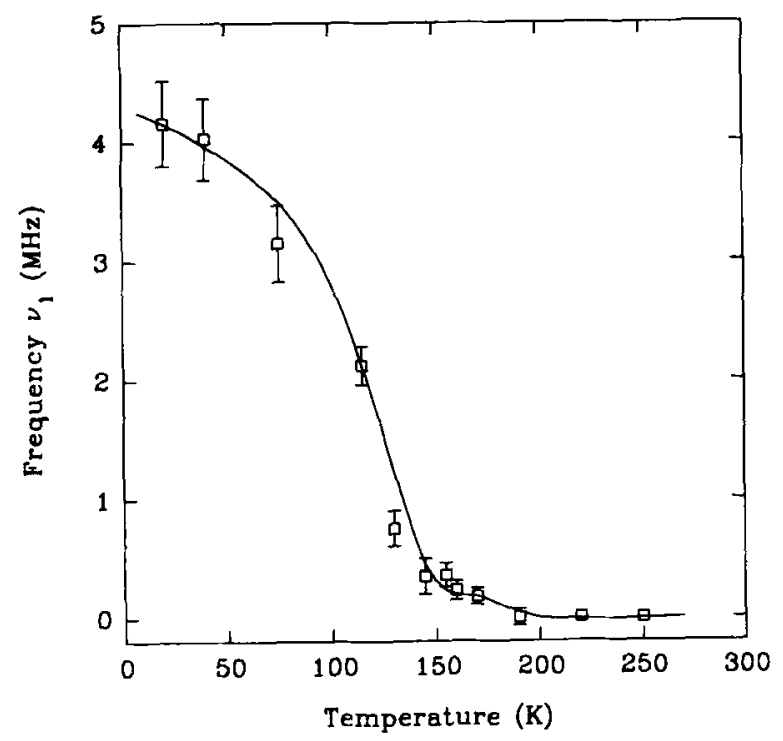

Fig. 3. Temperature evolution of the $\mathrm{ZF} \mu \mathrm{SR}$ frequency in $\mathrm{La}_{1.2} \mathrm{~Tb}_{0.8} \mathrm{CuO}_{4+\gamma}$. The solid line through the points is a guide to the eye.

\section{Discussion and conclusions}

Our results have thus shown that ordered local magnetic moments appear at the $\mathrm{Cu}^{+2}$ sites of $\mathrm{La}_{1.2} \mathrm{~Tb}_{0.8} \mathrm{CuO}_{4.05}$ below $170 \mathrm{~K}$, somewhat lower than the Néel temperatures of stoichiometric $\mathrm{La}_{2} \mathrm{CuO}_{4}$ and $\mathrm{Nd}_{2} \mathrm{CuO}_{4}$ $\left(\mathrm{T}_{N} \sim 250-280 \mathrm{~K}\right)$. The unit cell of the $\mathrm{T}^{*}$ phases is shown in Fig. 4; it arises through ordering of the supporting lanthanide cations, resulting in the fusion of half the $\mathrm{T}^{\prime}\left(\mathrm{Nd}_{2} \mathrm{CuO}_{4}\right.$, square planar $\mathrm{Cu}$ coordination) and half the $(\mathrm{O} / \mathrm{T})\left(\mathrm{La}_{2} \mathrm{CuO}_{4}\right.$, octahedral $\mathrm{Cu}$ coordination) unit cells. The magnetic structure of $\mathrm{Nd}_{2} \mathrm{CuO}_{4}[\vec{\mu}\|(1 / 2,1 / 2,0), \vec{\tau}\|(1 / 2,1 / 2,0)][8]$ is different from the one of $\mathrm{La}_{2} \mathrm{CuO}_{4}[\vec{\mu} \|(1 / 2,1 / 2,0), \vec{\tau} \perp(1 / 2,1 / 2,0)][9]$. Our neutron scattering results [7] for the present sample reveal a magnetic structure identical to $\mathrm{La}_{2} \mathrm{CuO}_{4}$ with a staggered moment at the $\mathrm{Cu}$ sites of $0.46(6) \mu_{B}$. The observed precession frequency at low temperatures corresponds to an internal field strength at the muon site of $\left\langle\mathrm{B}_{\mu}\right\rangle=$ $310(27) \mathrm{G}$. The temperature evolution of the average static field follows well a simple power law of the form $\left\langle\mathrm{B}_{\mu}\right\rangle=\left\langle\mathrm{B}_{\mu}(0)\right\rangle\left(1-\mathrm{T} / \mathrm{T}_{N}\right)^{\beta}$ with $\beta$ $=0.36(3)$ and $\mathrm{T}_{N}=146(1) \mathrm{K}$, supporting a $3 \mathrm{D}$ spin exchange Hamiltonian. We also performed exploratory dipolar field calculations of the local field at the muon site and the depolarisation rate in an attempt to locate the muon position in the unit cell. Using a magnetic moment of $\sim 0.5 \mu_{B}$ and the magnetic structure determined by neutron scattering, the most 


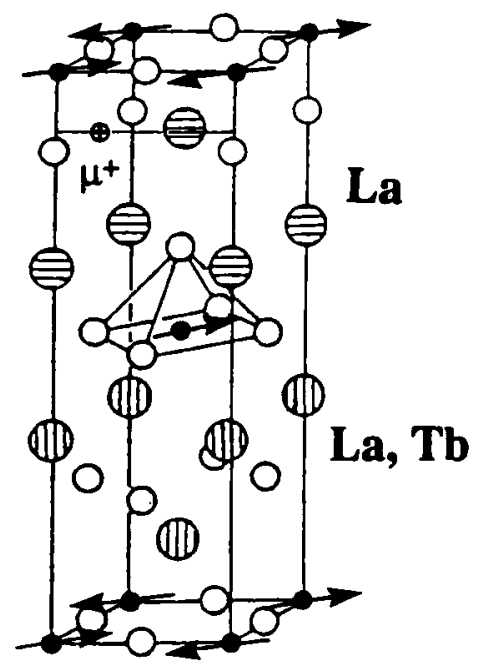

Fig. 4. The magnetic structure of $\mathrm{La}_{1.2} \mathrm{~Tb}_{0.8} \mathrm{CuO}_{4}$ and the probable muon site.

probable site is located on the face of the $(\mathrm{O} / \mathrm{T})$ part of the unit cell, $\sim 1 \AA$ away from the apical oxygen of the $\mathrm{CuO}_{5}$ square-pyramidal units [10].

At temperatures higher than $170 \mathrm{~K}$, the $\mu \mathrm{SR}$ signal does not display any oscillating features; moreover, long range magnetic order has now given way to an essentially temperature-independent Gaussian local field distribution with a width of $\left\langle\Delta \mathrm{B}^{2}\right\rangle^{1 / 2} \sim 23 \mathrm{G}$, presumably arising from randomly frozen copper moments which have lost their phase coherence due to thermal disorder. Measurements in a longitudinal field of $5 \mathrm{kG}$ confirm the static nature of this field distribution. However, the major contribution to the $\mu^{+} \mathrm{SR}$-spin depolarisation comes in this temperature regime from the Lorentzian component which is present throughout the temperature range of the experiment and whose fraction increases with increasing temperature. Relaxation of this hind is due to rapidly fluctuating internal field components perpendicular to the initial $\mu^{+}$polarisation and may be attributed to paramagnetic domains in the sample, that gradually grow in size with increasing temperature at the expense of magnetically ordered domains. A rough estimate of the spin fluctuation frequency may be derived from the measured damping constant $\lambda_{2} \sim 0.6(1) \mu \mathrm{s}^{-1}$ at low temperatures as $\nu \sim 10^{9} \mathrm{~Hz}$.

Finally, the abrupt increase in the depolarisation rate $\lambda_{1}$ below about $20 \mathrm{~K}$ corresponds to a substantially increased width of the internal field distribution to $\sim 650 \mathrm{G}$. This could be related to the onset of ordering of 
the $\mathrm{Tb}^{3+}$ sublattice. The lack of an oscillating component associated with this increased relaxation indicates that we may be witnessing short range order of the $\mathrm{Tb}^{3+}$ moments; this is in agreement with neutron scattering measurements at $1.5 \mathrm{~K}$ which fail to reveal additional magnetic Bragg peaks but show the presence of pronounced magnetic diffuse scattering [7], indicative of short range magnetic correlations. For a disordered $\mathrm{Tb}$ spin configuration, dipolar field calculations using the muon site determined earlier lead to relaxation rates in rough agreement with the observed values.

In conclusion, $\mu \mathrm{SR}$ measurements have revealed a rich magnetic behaviour of the parent $\mathrm{T}^{*}$ phase $\mathrm{La}_{1.2} \mathrm{~Tb}_{0.8} \mathrm{CuO}_{4}$. Static moments are present in the $\mathrm{Cu}$ sublattice below $280 \mathrm{~K}$ with long range order appearing in the vicinity of $170 \mathrm{~K}$. A decreasing volume fraction of the sample with decreasing temperature also exhibits rapid electronic spin fluctuations. Below $20 \mathrm{~K}$, short range magnetic order develops on the $\mathrm{Tb}$ sublattice.

\section{References}

[1] J.I. Budnick et al., Europhys. Lett. 5 (1988) 651.

[2] G.M. Luke et al., Nature 338 (1989) 49.

[3] M.J. Rosseinsky, K. Prassides, and C.A. Scott, Inorg. Chem. 30 (1991) 3367.

[4] R.F. Kiefl et al., Phys. Rev. Lett. 63 (1989) 2136; D.W. Cooke et al., Phys. Rev. B 41 (1990) 4801.

[5] N. Nishida et al., Physica C. 168 (1990) 23; B.J. Sternlieb et al., Phys. Rev. B 40 (1989) 11320.

[6] H. Sawa et al., Nature 337 (1989) 347.

[7] A. Lappas and K. Prassides, to be published.

[8] M.J. Rosseinsky, K. Prassides, and P. Day, J. Chem. Soc., Chem. Commun. (1989) 1734.

[9] D. Vaknin, S.K. Sinha, D.E. Moncton, D.C. Johnston, J.M. Newsam, C.R. Safinya, and H.E. King, Phys. Rev. Lett. 58 (1987) 2802.

[10] B. Hitti et al., Hyperfine Interactions 63 (1990) 287. 